\title{
Elegant Chaos in Fractional-Order System without Equilibria
}

\author{
Donato Cafagna and Giuseppe Grassi \\ Dipartimento Ingegneria Innovazione, Università del Salento, 73100 Lecce, Italy \\ Correspondence should be addressed to Donato Cafagna; donato.cafagna@unisalento.it
}

Received 16 July 2013; Accepted 30 November 2013

Academic Editor: Rafael Martínez-Guerra

Copyright (C) 2013 D. Cafagna and G. Grassi. This is an open access article distributed under the Creative Commons Attribution License, which permits unrestricted use, distribution, and reproduction in any medium, provided the original work is properly cited.

A new fractional-order chaotic system with no equilibria is presented. The proposed system can be considered elegant in the sense given by Sprott (2010), since the corresponding system equations contain very few terms and the system parameters have a minimum of digits. The chaotic dynamics are analyzed using the predictor-corrector algorithm when the fractional-order of the derivative is 0.98. Finally, the presence of chaos is validated by applying different numerical methods.

\section{Introduction}

Fractional calculus has been known since the early 17 th century, but its application to science and engineering has been developed only in recent years [1]. This happens because it has been recently found that several physical phenomena can be more accurately described by fractional differential equations and a number of numerical methods are available for approximating fractional derivatives [2-5]. Therefore, at present fractional calculus plays an important role in physics [2], electrical circuit theory [6], and control systems [7]. In particular, a significant role is played in chaos theory, where it has been shown that chaotic phenomena can be obtained in nonlinear dynamic systems with fractional order $[8,9]$. To this purpose, several chaotic fractional-order systems have been proposed starting from the chaotic integer-order counterparts. For example, by considering the pioneering Chua's circuit [10], some fractional-order counterparts have been proposed [1113]. Similarly, by considering the well-known integer-order Chen system [14], a number of fractional-order systems have been studied $[15,16]$. Moreover, the fractional Rössler system [17] and the fractional multiscroll system [18] have been developed starting from the corresponding integer-order systems $[19,20]$. Note that all the previous chaotic integerorder systems, along with their chaotic fractional-order counterpart, are characterized by one or more equilibrium points. On the other hand, very few papers have focused on the study of chaotic dynamics in integer-order and fractional-order systems without equilibria.

Referring to integer-order systems with no equilibria, the presence of chaos has been investigated only in [21-23]. In particular, in [21] a systematic search to find 3D integerorder chaotic systems with quadratic nonlinearities and no equilibria was performed. The objective was to find the algebraically simplest cases which cannot be further reduced by the removal of terms without destroying the chaos. Note that the presence of chaos in such systems is very surprising since they can have neither homoclinic nor heteroclinic orbits [24], and thus the Shilnikov method [24] cannot be used to verify the chaos. Referring to fractional-order systems, the study of chaotic systems with no equilibria is an almost unexplored topic, given that only one paper [25] has been published in literature to date.

Based on these considerations, in this paper, a new fractional-order chaotic system with no equilibrium points is presented. The proposed system, which represents the fractional-order counterpart of an integer-order system without equilibria studied in [21], can be considered "elegant" in the sense of Sprott [26] since the corresponding system equations contain no unnecessary terms and the system parameters have a minimum of digits. The presence of fractional chaos in the absence of equilibria has been validated by applying three different numerical methods when the system order is as low as 2.94 . 
The paper is organized as follows. In Section 2 the fundamentals of fractional derivative and the predictor-corrector method for solving fractional-order equations are illustrated. In Section 3 the equations of the considered fractional-order system with no equilibria are given. Moreover, the predictorcorrector algorithm is applied to solve the system and a chaotic attractor is found when the order of the derivative is $q=0.98$. In Section 4 three different numerical methods, including the recently introduced "0-1 test" for chaos [27], are applied to further confirm the presence of chaos in the proposed fractional-order chaotic system without equilibrium points. Finally, in Section 5 a brief discussion on the implementation of fractional-order circuit elements is reported.

\section{Basic Notions on Fractional Calculus and Derivatives}

The Riemann-Liouville fractional integral operator $J_{t_{0}}^{q}$ of order $q \in \mathfrak{R}^{+}$is defined on the Lebesque space $L_{1}\left[t_{0}, t_{1}\right]$ by

$$
J_{t_{0}}^{q} x(t) \triangleq \frac{1}{\Gamma(q)} \int_{t_{0}}^{t}(t-\tau)^{q-1} x(\tau) d \tau,
$$

where $\Gamma(q)$ is the Gamma function, with $J_{t_{0}}^{0} x(t)=x(t)$ [28].

For $\gamma>-1$ and $C$ being a real constant, two fundamental properties of the integral operator $J_{t_{0}}^{q}$ are [28]

$$
\begin{gathered}
J_{t_{0}}^{q}\left(t-t_{0}\right)^{\gamma}=\frac{\Gamma(\gamma+1)}{\Gamma(\gamma+1+q)}\left(t-t_{0}\right)^{\gamma+q}, \\
J_{t_{0}}^{q} C=C \frac{\Gamma(1)}{\Gamma(q+1)}\left(t-t_{0}\right)^{q}=\frac{C}{\Gamma(q+1)}\left(t-t_{0}\right)^{q} .
\end{gathered}
$$

Referring to the fractional differential operators, among the different definitions proposed in the literature, in this work the differential operator ${ }^{*} D_{t_{0}}^{q}$ proposed by Caputo is utilized:

$$
{ }^{*} D_{t_{0}}^{q} x(t) \triangleq J_{t_{0}}^{m-q} D_{t_{0}}^{m} x(t)=\frac{1}{\Gamma(m-q)} \int_{t_{0}}^{t} \frac{x^{(m)}(\tau)}{(t-\tau)^{q+1-m}} d \tau
$$

where $m-1<q \leq m$ and $m \in N$ (i.e., $m=\operatorname{ceil}(q))$ [29]. Two fundamental properties of the differential operator ${ }^{*} D_{t_{0}}^{q}$ are [28]

$$
\begin{aligned}
& { }^{*} D_{t_{0}}^{q} J_{t_{0}}^{q} x(t)=x(t), \\
& J_{t_{0}}^{q}\left({ }^{*} D_{t_{0}}^{q}\right) x(t)=J_{t_{0}}^{q} J_{t_{0}}^{m-q} D_{t_{0}}^{m} x(t)=J_{t_{0}}^{m} D_{t_{0}}^{m} x(t) \\
& =x(t)-\sum_{k=0}^{m-1} x^{(k)}\left(t_{0}^{+}\right) \frac{\left(t-t_{0}\right)^{k}}{k !} \text {. }
\end{aligned}
$$

Based on the Caputo's definition (3), consider the following general form of fractional-order differential equation:

$$
{ }^{*} D_{0, t}^{q} x(t)=f(x(t)), \quad x(0)=x_{0}, q \in(0,1) .
$$

The initial value problem (5) is equivalent to a Volterra integral equation [30],

$$
x(t)=x_{0}+\frac{1}{\Gamma(q)} \int_{0}^{t}(t-\tau)^{q-1} f(\tau, x(\tau)) d \tau,
$$

in the sense that a continuous function solves (6) if and only if it solves (5). Now, the predictor-corrector algorithm, which belongs to the family of Adams-Bashforth-Moulton schemes, is adopted to solve the Volterra integral equation (6). Firstly, the product trapezoidal quadrature formula is applied to replace the integral in (6). By taking $0 \leq t \leq T$ and by setting $h=T / N\left(N \in \mathbb{Z}^{+}\right), t_{n}=n h, n=0,1, \ldots, N,(6)$ can be discretized as [30]

$$
x\left(t_{n+1}\right)=x_{0}+\frac{h^{q}}{\Gamma(q+2)} \sum_{j=0}^{n+1} \alpha_{j, n+1} f\left(t_{j}, x\left(t_{j}\right)\right),
$$

where

$\alpha_{j, n+1}$

$$
= \begin{cases}n^{q+1}-(n-q)(n+1)^{q}, & j=0 \\ (n-j+2)^{q+1}+(n-j)^{q+1}-2(n-j+1)^{q+1}, & 1 \leq j \leq n \\ 1 & j=n+1 .\end{cases}
$$

Equation (7) can be rewritten as [30]

$$
\begin{aligned}
x\left(t_{n+1}\right)= & x_{0}+\frac{h^{q}}{\Gamma(q+2)} f\left(t_{n+1}, x\left(t_{n+1}\right)\right) \\
& +\frac{h^{q}}{\Gamma(q+2)} \sum_{j=0}^{n} \alpha_{j, n+1} f\left(t_{j}, x\left(t_{j}\right)\right) .
\end{aligned}
$$

In order to start the Adams-Moulton iterative method, the solution is accomplished by first predicting $\left(x^{(p)}\left(t_{n+1}\right)\right)$ via the explicit Adams-Bashforth formula and then correcting $\left(x\left(t_{n+1}\right)\right)$. Thus, (9) is solved as [30]

$$
\begin{aligned}
x^{(p)}\left(t_{n+1}\right) & =x_{0}+\frac{1}{\Gamma(q)} \sum_{j=0}^{n} \beta_{j, n+1} f\left(t_{j}, x\left(t_{j}\right)\right), \\
x\left(t_{n+1}\right)= & x_{0}+\frac{h^{q}}{\Gamma(q+2)} f\left(t_{n+1}, x^{(p)}\left(t_{n+1}\right)\right) \\
& +\frac{h^{q}}{\Gamma(q+2)} \sum_{j=0}^{n} \alpha_{j, n+1} f\left(t_{j}, x\left(t_{j}\right)\right),
\end{aligned}
$$

where

$$
\beta_{j, n+1}=\frac{h^{q}}{q}\left((n+1-j)^{q}-(n-j)^{q}\right), \quad 0 \leq j \leq n .
$$

The error estimate of the algorithm is $e=\operatorname{Max} \mid x\left(t_{j}\right)-$ $x_{h}\left(t_{j}\right) \mid=O\left(h^{\rho}\right)(j=0,1, \ldots, N)$, where $\rho=\operatorname{Min}(2,1+q)$ [31].

Finally, attention is focused on the existence of periodic solutions in fractional-order systems [32-34]. In this 
regard, in [32] Tavazoei and Haeri have analytically proved that, different from integer-order linear/nonlinear systems, fractional-order systems cannot generate exact periodic solutions. In other words, the theorem proved in [32] states that the fractional-order time-invariant system (5) defined via the Caputo derivative cannot have any nonconstant smooth periodic solution.

\section{Elegant Fractional-Order Chaotic System with No Equilibria}

In [21] a systematic search to find three-dimensional integerorder chaotic systems with quadratic nonlinearities and no equilibria was performed. The objective was to find the algebraically simplest cases which cannot be further reduced by the removal of terms without destroying the chaos. In particular, in [21] attention is focused on the class of integer-order chaotic systems described by equations

$$
\begin{gathered}
\dot{x}=y, \quad \dot{y}=z, \\
\dot{z}=k_{1} x+k_{2} y+k_{3} z+k_{4} x^{2}+k_{5} y^{2}+k_{6} z^{2} \\
+k_{7} x y+k_{8} x z+k_{9} y z+a
\end{gathered}
$$

with the condition $k_{1}^{2}-4 k_{4} a \leq 0$. Note that a general requirement for chaotic systems (12) is the presence of a constant term, since there would otherwise be at least one equilibrium point at the origin $(0,0,0)$. For each case analyzed in [21], the space of coefficients was searched for values that are deemed "elegant" [26], meaning that as many coefficients as possible are set to zero with the others being set to \pm 1 if possible or otherwise to a small integer or decimal fraction with the fewest possible digits. Based on these considerations, by taking the parameters $k_{1}=0, k_{2}=-1, k_{3}=0, k_{4}=0$, $k_{5}=0, k_{6}=0, k_{7}=0, k_{8}=-1$, and $k_{9}=-1$, the following "elegant" integer-order chaotic system, listed as NE6 in [21], is obtained:

$$
\begin{gathered}
\dot{x}=y, \quad \dot{y}=z, \\
\dot{z}=-y-x z-y z+a .
\end{gathered}
$$

As shown in [21] system (13) with $a=-0.75$ has no equilibria and exhibits the attractor reported in Figure 1, with the Lyapunov exponents given by $\lambda_{1}=0.0280, \lambda_{2}=0$, and $\lambda_{3}=-3.4341$. Note that the presence of chaos in such system is very surprising since it can have neither homoclinic nor heteroclinic orbits [24], and thus the Shilnikov method [24] cannot be used to verify the chaos.

Based on previous considerations, attention is now focused on the fractional-order counterpart of system (13). Namely, the equations of the proposed elegant fractionalorder system are

$$
\begin{aligned}
& { }^{*} D^{q} x=y, \quad{ }^{*} D^{q} y=z, \\
& { }^{*} D^{q} z=-y-x z-y z+a,
\end{aligned}
$$

where ${ }^{*} D^{q}$ denotes the Caputo fractional operator defined in (3) with initial time $t_{0}=0$, order $q \in(0,1)$, and $a \neq 0$ [29].

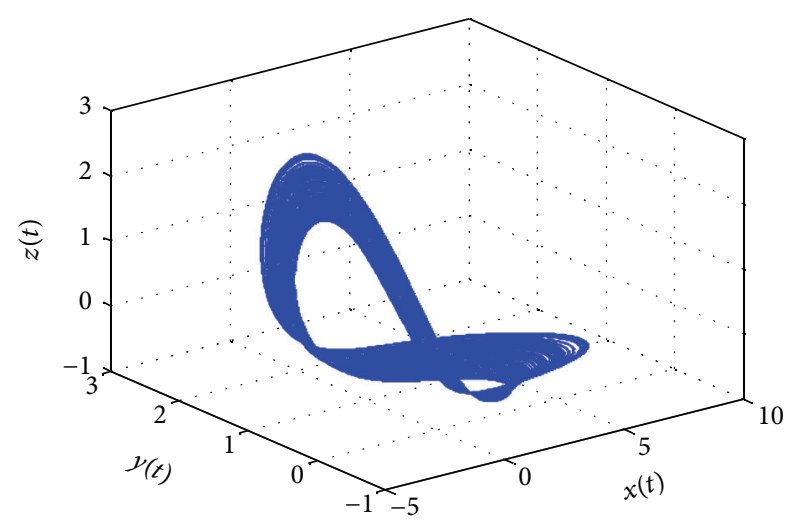

FIgURE 1: Integer-order chaotic attractor in the space $(x, y, z)$ of system (13) with $a=-0.75$ and initial conditions $(0,3,-0.1)$.

It can be readily verified that the proposed system (14) has no equilibrium points.

Now, by applying the predictor-corrector algorithm described in Section 2, the solution of the fractional system (14) can be written as

$$
\begin{aligned}
& x_{h}\left(t_{n+1}\right)= x_{0}+\frac{h^{q}}{\Gamma(q+2)}\left(y^{(p)}\left(t_{n+1}\right)\right) \\
&+\frac{h^{q}}{\Gamma(q+2)} \sum_{j=0}^{n} \alpha_{j, n+1}\left(y\left(t_{j}\right)\right), \\
& y_{h}\left(t_{n+1}\right)= y_{0}+\frac{h^{q}}{\Gamma(q+2)}\left(z^{(p)}\left(t_{n+1}\right)\right) \\
&+\frac{h^{q}}{\Gamma(q+2)} \sum_{j=0}^{n} \alpha_{j, n+1}\left(z\left(t_{j}\right)\right), \\
& z_{h}\left(t_{n+1}\right)= z_{0}+\frac{h^{q}}{\Gamma(q+2)}\left(-y^{(p)}\left(t_{n+1}\right)\right. \\
&+\frac{h^{q}}{\Gamma(q+2)} \sum_{j=0}^{n} \alpha_{j, n+1}\left(-y\left(t_{j}\right)-x\left(t_{j}\right) z\left(t_{j}\right)\right. \\
&\left.-y^{(p)}\left(t_{n+1}\right) z^{(p)}\left(t_{n+1}\right) z^{(p)}\left(t_{n+1}\right)+a\right) \\
&\left.-y\left(t_{j}\right) z\left(t_{j}\right)+a\right)
\end{aligned}
$$

in which the predicted variables are

$$
\begin{aligned}
& x^{(p)}\left(t_{n+1}\right)=x_{0}+\frac{1}{\Gamma(q)} \sum_{j=0}^{n} \beta_{j, n+1}\left(y\left(t_{j}\right)\right), \\
& y^{(p)}\left(t_{n+1}\right)=y_{0}+\frac{1}{\Gamma(q)} \sum_{j=0}^{n} \beta_{j, n+1}\left(z\left(t_{j}\right)\right),
\end{aligned}
$$




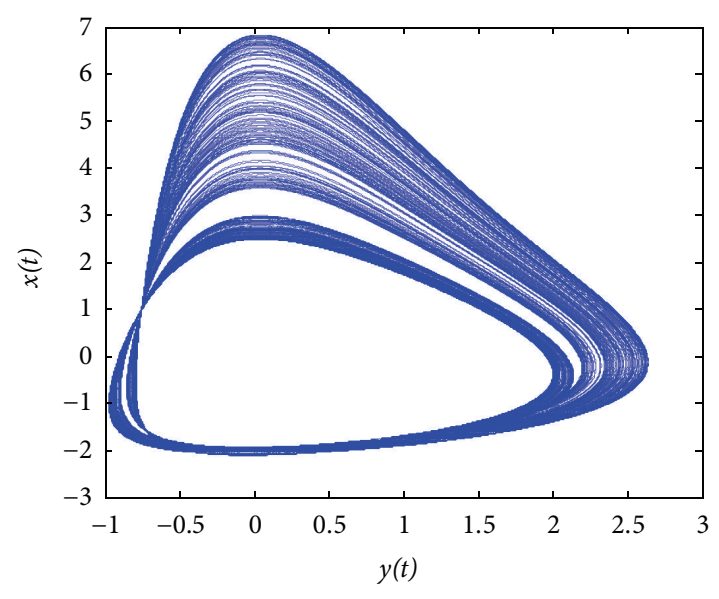

(a)

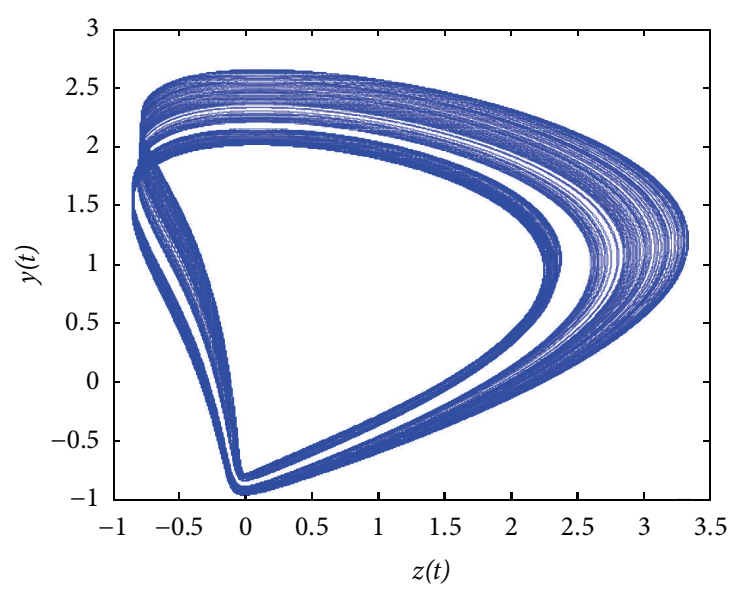

(b)

FIGURE 2: Chaotic attractor of the elegant fractional-order system without equilibrium point (14) with $q=0.98, a=-0.757$ and initial conditions $(0,3,-0.1)$ : (a) chaotic attractor in the $(y, x)$-plane, (b) chaotic attractor in the $(z, y)$-plane.

$$
\begin{array}{r}
z^{(p)}\left(t_{n+1}\right) \\
=z_{0}+\frac{1}{\Gamma(q)} \sum_{j=0}^{n} \beta_{j, n+1}\left(-y\left(t_{j}\right)-x\left(t_{j}\right) z\left(t_{j}\right)\right. \\
\left.-y\left(t_{j}\right) z\left(t_{j}\right)+a\right),
\end{array}
$$

where $\alpha_{j, n+1}$ and $\beta_{j, n+1}$ are given by (8) and (11), respectively. The discretized equations (15)-(16) are calculated for several values of order $0<q<1$ and parameter $a$. A remarkable finding of this paper is that chaos exists in the proposed elegant fractional-order system without equilibrium points. In particular, by setting $a=-0.757$ and by varying order $q$, it has been found that chaos exists in the fractional-order system (14) for $q=0.98$. The phase portraits of the chaotic attractor are shown in Figure 2.

\section{Tests for Chaos and Dynamic Behaviors}

The chaotic nature of the attractor reported in Figure 2 is validated using different approaches.

The first approach is based on the reliable and efficient binary test for chaos, called 0-1 test, which has been recently proposed and applied to fractional-order systems in [27]. The idea underlying the test is to construct a random walktype process from the discrete data (sampled at times $n=$ $1,2, \ldots, N)$ and then to examine how the variance of the random walk scales with time [27]. Specifically, the method is based on the computation of the asymptotic growth rate $K$ : when $K$ is close to 0 , the motion is classified as regular (i.e., periodic or quasi-periodic); when $K$ is close to 1 , the motion is classified as chaotic [27]. The value of $K$, obtained from (15)-(16), asymptotically approaches 1 for $q=0.98$ and $a=$ -0.757 , indicating that the dynamics of system (14) are chaotic (Figure 3).

The second approach is based on the recent paper by Caponetto and Fazzino [35]. In such paper, an application

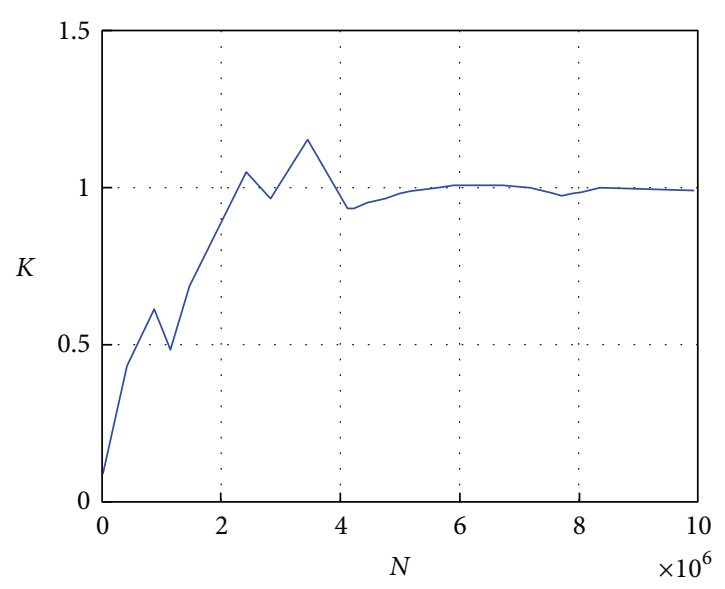

FIgURE 3: The 0-1 test for chaos when $q=0.98, a=-0.757$ : dependence of the asymptotic growth rate $K$ on the length $N$ of the time series (15)-(16).

of a well-known transformation technique (i.e., the differential transform method) to fractional differential equations is employed for calculating the Lyapunov exponents. The obtained spectrum $\left(\lambda_{1}=0.011, \lambda_{2}=0\right.$, and $\left.\lambda_{3}=-3.025\right)$ is still characterized by a positive value.

The last approach consists in computing the maximum Lyapunov exponent by Wolf algorithm [36]. The computed value $\left(\lambda_{\max }=0.021\right)$ is positive, confirming the chaotic behaviour of the considered fractional-order system. Note that such value is consistent with the maximum Lyapunov exponent values of all the integer-order chaotic systems with no equilibria analyzed by Jafari et al. in [21].

Now, the objective is to investigate the system behavior by setting $q=0.98$ and by varying parameter $a$ within a neighborhood of the value $a=-0.757$. In particular, for $a=$ -0.74 the system trajectories go toward infinity, whereas for $a=-0.759$ fractional system (14) possesses the nonchaotic behavior reported in Figure 4. 


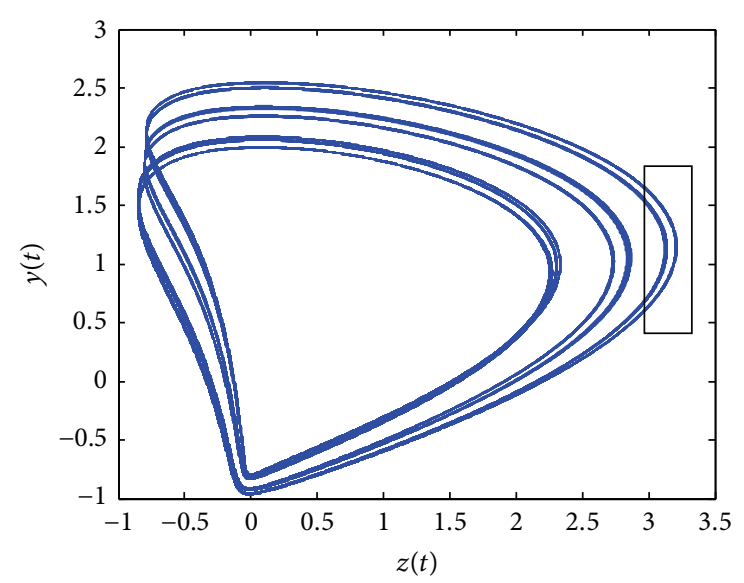

(a)

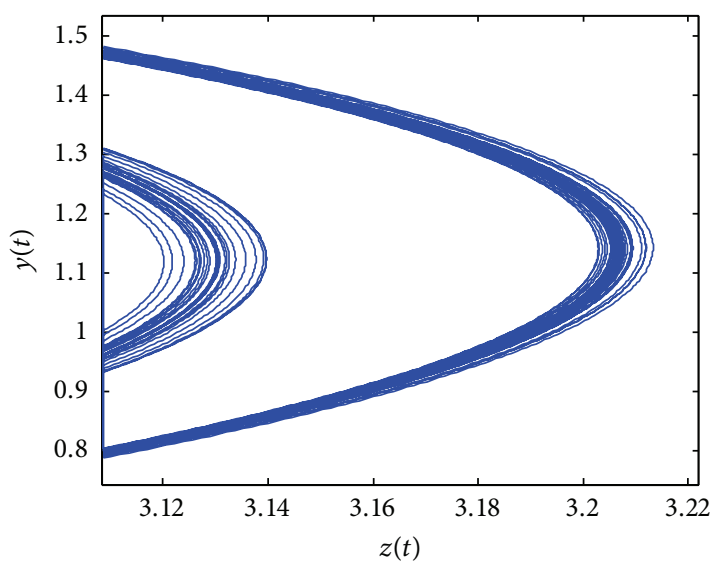

(b)

FIGURE 4: Nonchaotic behaviour of the fractional system (14) for $q=0.98$ and $a=-0.759$ : (a) oscillations plotted in the ( $z$, $y$ )-plane; (b) zoom in on the system trajectories, indicating that they never return on the same points in the state space.

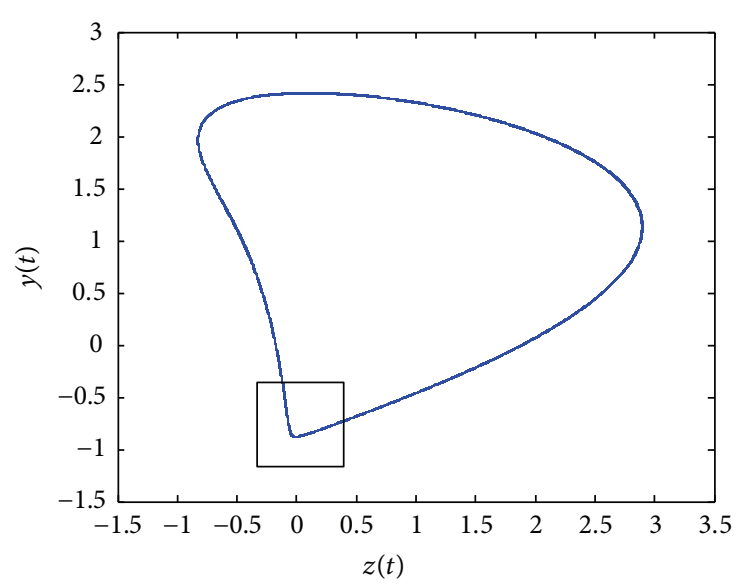

(a)

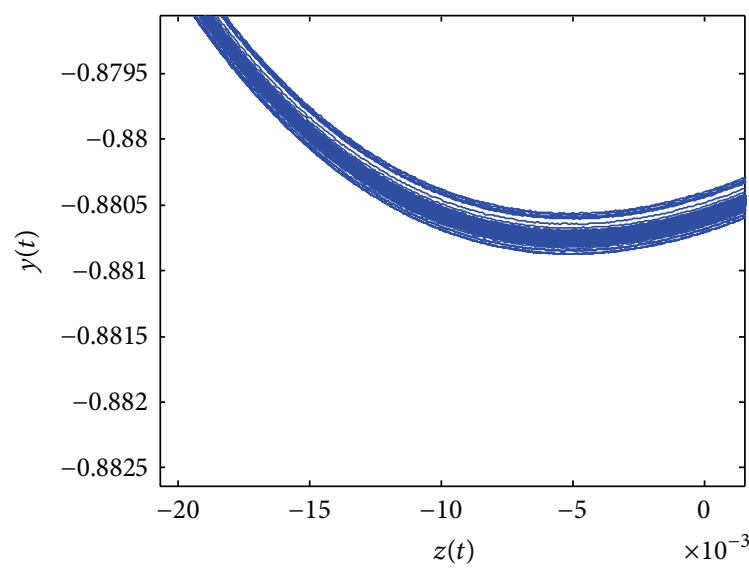

(b)

FIGURE 5: Nonchaotic behaviour of the fractional system (14) for $q=0.98$ and $a=-0.80$ : (a) oscillations plotted in the $(z, y)$-plane; (b) zoom in on the system trajectories.

Notice that, although the trajectories shown in Figure 4(a) seem to be periodic oscillations, in Section 2 it has been stated that such oscillations are not exactly periodic. Figure 4(b) shows that the system trajectory never returns on the same points in the state space, confirming that such oscillations are not exactly periodic. Such nonchaotic behavior, characterized by oscillations not exactly periodic, is also found for value $a=-0.80$, as shown in Figure 5.

\section{Discussion on the Implementation of Fractional-Order Circuit Elements}

The aim of this section is to provide some considerations on the practical implementation of the proposed fractionalorder system (14). In particular, by considering the three equations in (14), it is clear that the challenge is the implementation of circuit elements that realize the fractional derivatives ${ }^{*} D^{q}$, given that the remaining terms in (14) can be realized using standard circuit elements (such as resistors, analog multipliers, and operational amplifiers) [37,38]. First of all, by following the common practice, the dimensionless variables $x, y$, and $z$ in (14) are properly scaled and assumed to be electrical voltages. Thus, the Laplace transform applied to the fractional derivative ${ }^{*} D^{q}$ of the scaled variables leads to the following relationship between the current and the voltage across the fractional capacitor:

$$
V(s)=\frac{1}{s^{q} C_{F}} I(s),
$$

where $C_{F}$ is the value of the fractional capacitance [5]. Note that in (17) the phase difference between the current and voltage of the capacitor is $q(\pi / 2)$, which represents a generalization of the well-known phase difference $\pi / 2$ when the capacitor is of integer order $(q=1)$. The mathematical approximation of the equivalent transfer function $\left(1 / s^{q}\right)(0<$ $q<1$ ), within a certain region of frequencies, has been 


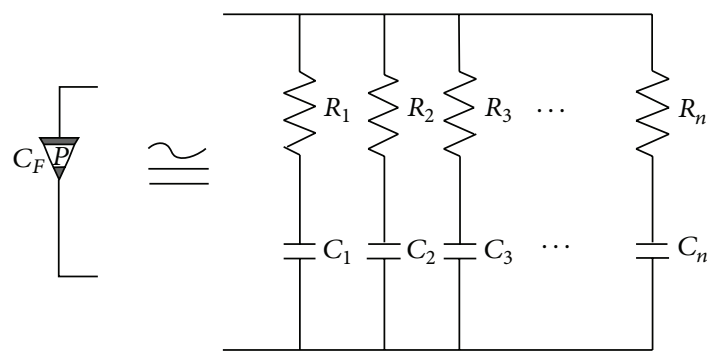

FIgURE 6: An example of circuit implementation of a fractional capacitor $C_{F}$.

realized in the literature using several approaches, which involve the adoption of a number of resistors and capacitors connected via different topologies [5]. An example of circuit implementation of a fractional capacitor $C_{F}$ is shown in Figure 6. Recently, new realizations of fractional-order capacitors have been proposed using chemical reactions between different materials [39, 40].

Note that such considerations related to the practical realization of fractional-order elements have been recently exploited in the literature for successfully implementing some examples of fractional-order chaotic circuits [38, 41]. Based on these encouraging results, the authors have planned to start implementing system (14) in the near future.

\section{Conclusions}

The presence of chaos in both integer-order and fractionalorder systems without equilibria represents a new exciting phenomenon and an almost unexplored field of research. Note that, since these systems can have neither homoclinic nor heteroclinic orbits, the Shilnikov method cannot be used to demonstrate the presence of chaos. In this paper a new fractional-order system with no equilibrium points has been presented. The proposed system, which can be considered elegant in the sense given by Sprott, has shown chaotic behavior when the system order is as low as 2.94. The system dynamics have been analyzed using the predictor-corrector algorithm and the presence of chaos has been validated by applying three different methods.

\section{References}

[1] D. Cafagna, "Fractional calculus: a mathematical tool from the past for present engineers," IEEE Industrial Electronics Magazine, vol. 1, no. 2, pp. 35-40, 2007.

[2] J. A. Tenreiro Machado, M. F. Silva, R. S. Barbosa et al., "Some applications of fractional calculus in engineering," Mathematical Problems in Engineering, vol. 2010, Article ID 639801, 34 pages, 2010.

[3] H. Sun, A. Abdelwahab, and B. Onaral, "Linear approximation for transfer function with a pole of fractional order," IEEE Transactions on Automatic Control, vol. 29, no. 5, pp. 441-444, 1984.

[4] K. Diethelm, N. J. Ford, and A. D. Freed, "A predictor-corrector approach for the numerical solution of fractional differential equations," Nonlinear Dynamics, vol. 29, no. 1-4, pp. 3-22, 2002.
[5] I. Podlubny, Fractional Differential Equations, vol. 198, Academic Press, New York, NY, USA, 1999.

[6] P. Arena, R. Caponetto, L. Fortuna, and D. Porto, Nonlinear Noninteger Order Circuits and Systems-An Introduction, vol. 38, World Scientific, Singapore, 2000.

[7] M. Rivero, S. V. Rogosin, J. A. Tenreiro Machado, and J. J. Trujillo, "Stability of fractional order systems," Mathematical Problems in Engineering, vol. 2013, Article ID 356215, 14 pages, 2013.

[8] M. S. Abd-Elouahab, N.-E. Hamri, and J. Wang, "Chaos control of a fractional-order financial system," Mathematical Problems in Engineering, vol. 2010, Article ID 270646, 18 pages, 2010.

[9] C. M. A. Pinto and J. A. Tenreiro Machado, "Complex order van der Pol oscillator," Nonlinear Dynamics, vol. 65, no. 3, pp. 247254, 2011.

[10] L. O. Chua, M. Komuro, and T. Matsumoto, "The double scroll family," IEEE Transactions on Circuits and Systems I, vol. 33, no. 11, pp. 1072-1118, 1986.

[11] T. Hartley, C. Lorenzo, and H. Qammer, "Chaos in a fractional order Chua's system," IEEE Transactions on Circuits and Systems I, vol. 42, no. 8, pp. 485-490, 1995.

[12] C. P. Li, W. H. Deng, and D. Xu, "Chaos synchronization of the Chua system with a fractional order," Physica A, vol. 360, no. 2, pp. 171-185, 2006.

[13] D. Cafagna and G. Grassi, "Fractional-order Chua's circuit: time-domain analysis, bifurcation, chaotic behavior and test for chaos," International Journal of Bifurcation and Chaos in Applied Sciences and Engineering, vol. 18, no. 3, pp. 615-639, 2008.

[14] G. Chen and T. Ueta, "Yet another chaotic attractor," International Journal of Bifurcation and Chaos in Applied Sciences and Engineering, vol. 9, no. 7, pp. 1465-1466, 1999.

[15] J. G. Lu and G. Chen, "A note on the fractional-order Chen system," Chaos, Solitons and Fractals, vol. 27, no. 3, pp. 685-688, 2006.

[16] D. Cafagna and G. Grassi, "Bifurcation and chaos in the fractional-order Chen system via a time-domain approach," International Journal of Bifurcation and Chaos, vol. 18, no. 7, pp. 1845-1863, 2008.

[17] D. Cafagna and G. Grassi, "Hyperchaos in the fractional-order Rössler system with lowest-order," International Journal of Bifurcation and Chaos, vol. 19, no. 1, pp. 339-347, 2009.

[18] W. Deng and J. Lü, "Design of multidirectional multiscroll chaotic attractors based on fractional differential systems via switching control," Chaos, vol. 16, no. 4, Article ID 043120, 2006.

[19] O. E. Rössler, "An equation for hyperchaos," Physics Letters A, vol. 71, no. 2-3, pp. 155-157, 1979.

[20] D. Cafagna and G. Grassi, "Hyperchaotic coupled Chua circuits: an approach for generating new nxm-scroll attractors," International Journal of Bifurcation and Chaos, vol. 13, no. 9, pp. 25372550, 2003.

[21] S. Jafari, J. C. Sprott, and S. M. R. Hashemi Golpayegani, "Elementary quadratic chaotic flows with no equilibria," Physics Letters A, vol. 377, no. 9, pp. 699-702, 2013.

[22] Z. Wei, "Dynamical behaviors of a chaotic system with no equilibria," Physics Letters A, vol. 376, no. 2, pp. 102-108, 2011.

[23] Z. Wang, S. Cang, E. O. Ochola, and Y. Sun, "A hyperchaotic system without equilibrium," Nonlinear Dynamics, vol. 69, no. 1-2, pp. 531-537, 2012.

[24] J. Guckenheimer and P. Holmes, Nonlinear Oscillations, Dynamical Systems, and Bifurcations of Vector Fields, vol. 42, Springer, New York, NY, USA, 1983. 
[25] H. Li, X. Liao, and M. Luo, "A novel non-equilibrium fractionalorder chaotic system and its complete synchronization by circuit implementation," Nonlinear Dynamics, vol. 68, no. 1-2, pp. 137-149, 2012.

[26] J. C. Sprott, Elegant Chaos: Algebraically Simple Chaotic Flows, World Scientific, Singapore, 2010.

[27] D. Cafagna and G. Grassi, "An effective method for detecting chaos in fractional-order systems," International Journal of Bifurcation and Chaos, vol. 20, no. 3, pp. 669-678, 2010.

[28] R. Gorenflo and F. Mainardi, "Fractional calculus: integral and differential equations of fractional order," in Fractals and Fractional Calculus in Continuum Mechanics, vol. 378, Springer, Vienna, Austria, 1997.

[29] M. Caputo, "Linear models of dissipation whose Q is almost frequency independent. Part II," Geophysical Journal of the Royal Astronomical Society, vol. 13, pp. 529-539, 1967.

[30] K. Diethelm and N. J. Ford, "Analysis of fractional differential equations," Journal of Mathematical Analysis and Applications, vol. 265, no. 2, pp. 229-248, 2002.

[31] K. Diethelm, N. J. Ford, and A. D. Freed, "Detailed error analysis for a fractional Adams method," Numerical Algorithms, vol. 36, no. 1, pp. 31-52, 2004.

[32] M. S. Tavazoei and M. Haeri, "A proof for non existence of periodic solutions in time invariant fractional order systems," Automatica, vol. 45, no. 8, pp. 1886-1890, 2009.

[33] M. S. Tavazoei, "A note on fractional-order derivatives of periodic functions," Automatica, vol. 46, no. 5, pp. 945-948, 2010.

[34] M. Yazdani and H. Salarieh, "On the existence of periodic solutions in time-invariant fractional order systems," Automatica, vol. 47, no. 8, pp. 1834-1837, 2011.

[35] R. Caponetto and S. Fazzino, "A semi-analytical method for the computation of the Lyapunov exponents of fractional-order systems," Communications in Nonlinear Science and Numerical Simulation, vol. 18, no. 1, pp. 22-27, 2013.

[36] A. Wolf, J. B. Swift, H. L. Swinney, and J. A. Vastano, "Determining Lyapunov exponents from a time series," Physica D, vol. 16, no. 3, pp. 285-317, 1985.

[37] A. S. Elwakil, "Fractional-order circuits and systems: an emerging interdisciplinary research area," IEEE Circuits and Systems Magazine, vol. 10, no. 4, pp. 40-50, 2010.

[38] C.-X. Liu and L. Liu, "Circuit implementation of a new hyperchaos in fractional-order system," Chinese Physics B, vol. 17, no. 8, pp. 2829-2836, 2008.

[39] K. Biswas, S. Sen, and P. K. Dutta, "Modeling of a capacitive probe in a polarizable medium," Sensors and Actuators A, vol. 120, no. 1, pp. 115-122, 2005.

[40] I. S. Jesus and J. A. Machado, "Development of fractional order capacitors based on electrolyte processes," Nonlinear Dynamics, vol. 56, no. 1-2, pp. 45-55, 2009.

[41] D. Zhu, C. X. Liu, and B. Yan, "Drive-response synchronization of a fractional-order hyperchaotic system and its circuit implementation," Mathematical Problems in Engineering, vol. 2013, Article ID 815765, 8 pages, 2013. 


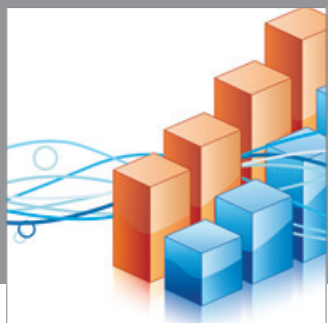

Advances in

Operations Research

mansans

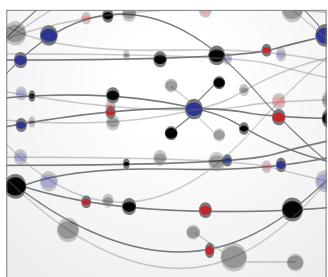

The Scientific World Journal
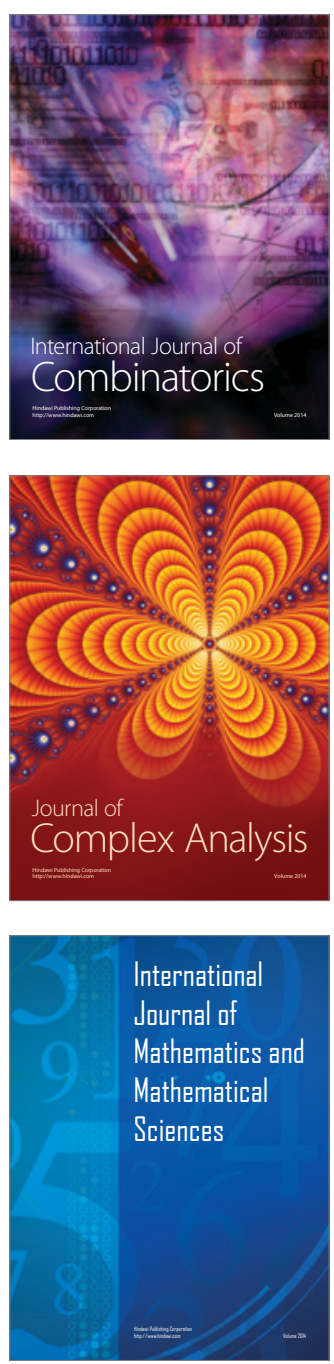
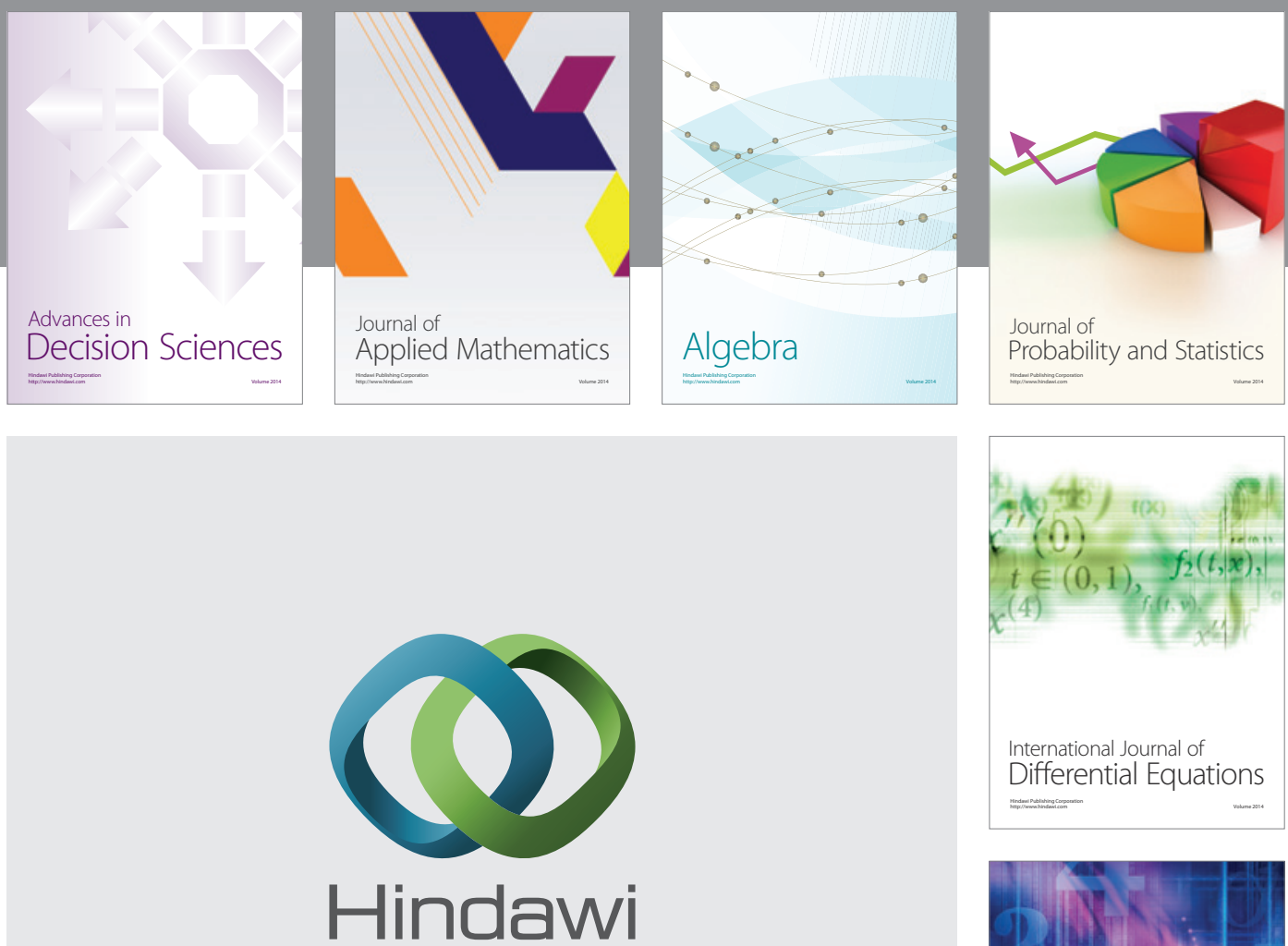

Submit your manuscripts at http://www.hindawi.com
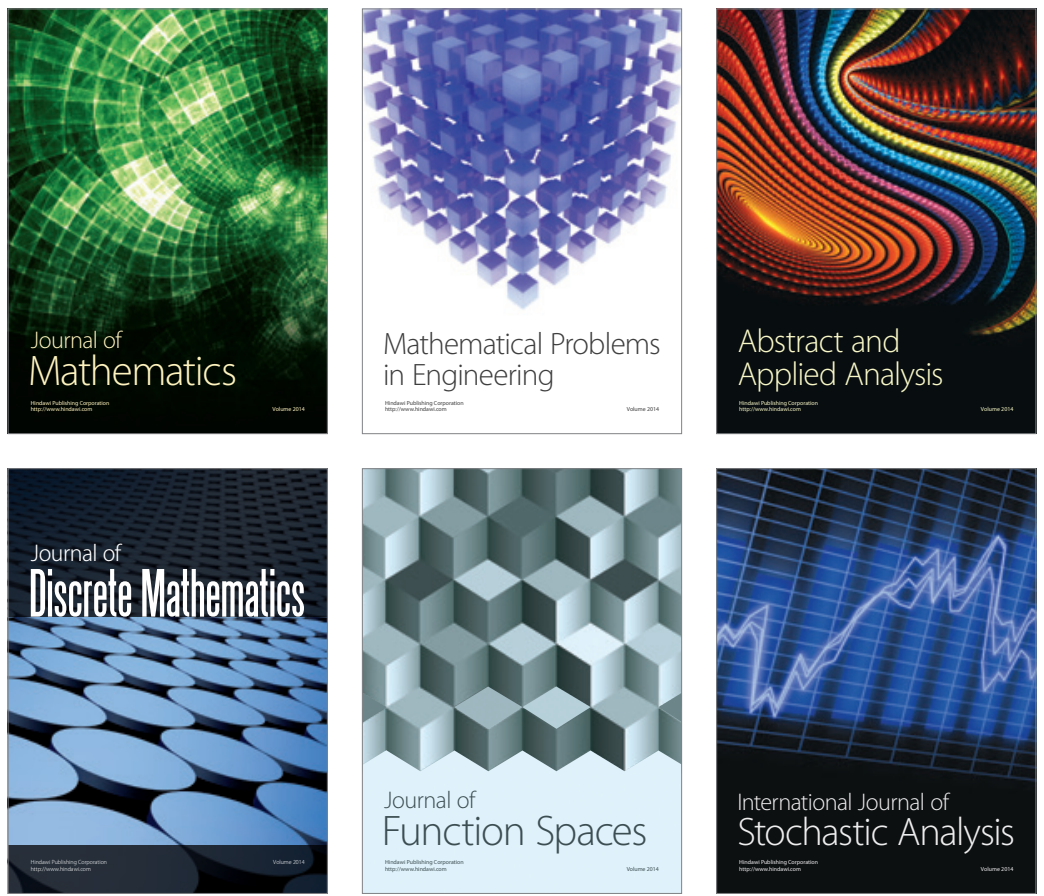

Journal of

Function Spaces

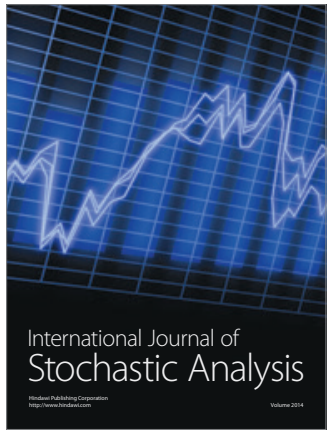

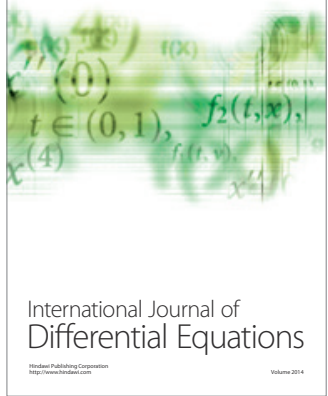
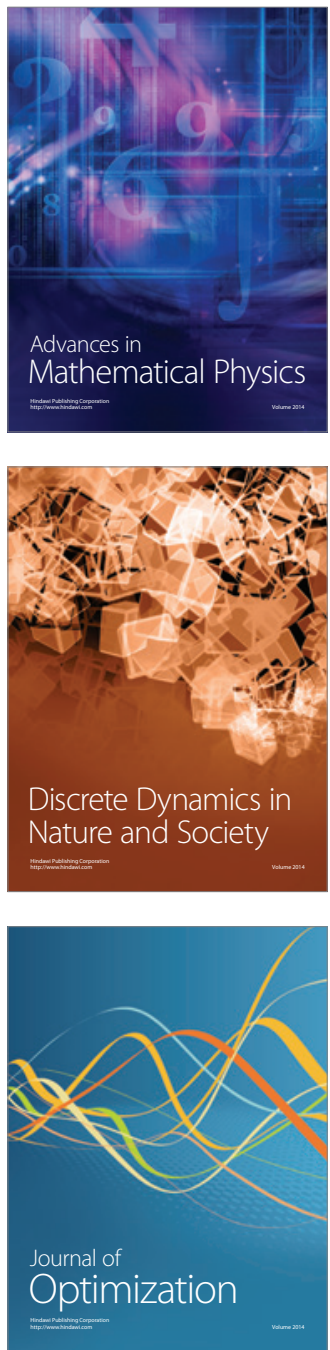